\title{
First-Time Patellofemoral Dislocation: Risk Factors for Recurrent Instability
}

\author{
Laura Lewallen, $\mathrm{MD}^{1} \quad$ Amy McIntosh, $\mathrm{MD}^{1} \quad$ Diane Dahm, $\mathrm{MD}^{1}$ \\ 1 Department of Orthopedic Surgery, Mayo Clinic, \\ Address for correspondence Diane Dahm, MD, Mayo Clinic, 200 First \\ Rochester, Minnesota \\ Street SW, Rochester, MN 55905 (e-mail: dahm.diane@mayo.edu).
}

J Knee Surg 2015;28:303-310.

\begin{abstract}
Keywords

- patellofemoral dislocation

- patellar instability

Patellofemoral instability is a complex problem, which can be difficult to manage. The purpose of this study was to describe the demographics of patients with a first-time patellofemoral dislocation, and identify risk factors for recurrent instability. This was a single institution, institutional review board-approved, retrospective review of $>2,000$ patients with a patellar dislocation between 1998 and 2010. Inclusion criteria are as follows: (1) no prior history of patellofemoral subluxation or dislocation of the affected knee; (2) X-rays within 4 weeks of the initial instability episode; and (3) a dislocated patella requiring reduction, or history/findings suggestive of acute patellar dislocation (effusion/hemarthrosis, tenderness along the medial parapatellar structures, and apprehension with lateral patellar translation). Clinical records and radiographs were reviewed. The Caton-Deschamps and Insall-Salvati indices were used to evaluate patella alta. Trochlear dysplasia was assessed using the Dejour classification system. Skeletal maturity was graded based on the distal femoral and proximal tibial physes, using one of the following categories: open, closing, or closed. Three hundred twenty-six knees (312 patients) met the aforementioned criteria. There were 145 females (46.5\%) and 167 males (53.5\%), with an average age of 19.6 years (range, 9-62 years). Thirty-five patients (10.7\%) were treated with surgery after the initial dislocation. All others were initially managed nonoperatively. Of the 291 patients managed nonoperatively, 89 (30.6\%) had recurrent instability, 44 (49.4\%) of which eventually required surgery. Several risk factors for recurrent instability were identified, including younger age $(p<0.01)$, immature physes $(p<0.01)$, sports-related injuries $(p<0.01)$, patella alta $(p=0.02)$, and trochlear dysplasia $(p<0.01)$. Sixty-nine percent of patients with a firsttime patellofemoral dislocation will stabilize with conservative treatment. However, patients younger than 25 years with trochlear dysplasia have a 60 to $70 \%$ risk of recurrence by 5 years. This information is helpful when counseling patients on their risk for recurrent instability and determining the most appropriate treatment plan. The clinical tool shown in Fig. 4 may be especially useful.
\end{abstract}

Patellofemoral instability is a complex problem, which can be difficult to manage. While most common among the adolescent age group,,$^{1-3}$ the annual incidence of acute patellar dislocation in the general population is estimated between
5.8 and 43 per $100,000 .^{3-5}$ These isolated injuries can have lasting consequences, such as pain, recurrent instability, decreased activity level, and potentially patellofemoral arthritis. $2,6,7$ received

October 27, 2014

accepted after revision

November 17, 2014

published online

January 29, 2015
Copyright $\odot 2015$ by Thieme Medical Publishers, Inc., 333 Seventh Avenue, New York, NY 10001, USA. Tel: +1(212) 584-4662.
DOI http://dx.doi.org/ 10.1055/s-0034-1398373. ISSN $1538-8506$. 
Several epidemiologic, anatomic, and radiographic risk factors for patellofemoral instability have been identified and described by various authors., ${ }^{2,7,8}$ The classic characteristics include female gender, adolescence, and obesity. However, these risk factors have been questioned by several authors in recent years due to concerns for sampling bias given limitations of study design. ${ }^{1,9}$

Despite the relative frequency of these acute injuries, as well as the substantial body of literature on the topic, the prognosis for many of these patients remains uncertain. It is difficult to predict which patients will go on to have recurrent instability; therefore, the most appropriate treatment method is not always apparent at the time of the initial injury. The increasing number of options for surgical management can also complicate the treatment plan.

The majority of patients with a first-time dislocation are managed nonoperatively; however, a significant number of these patients have future problems with instability. According to prior studies, approximately $44 \%$ will have recurrent dislocations, ${ }^{6,7,10,11}$ and nearly half ${ }^{7,12}$ will have recurrent instability or pain after nonoperative treatment. A detailed description of the demographics of these patients would provide valuable information which would likely influence management.

This study was undertaken to describe the demographics of a large cohort of patients with acute, first-time patellofemoral dislocation. The goal was to identify risk factors for recurrent instability and allow for a more accurate assessment of patients at the time of injury, to determine the most appropriate treatment plan.

\section{Materials and Methods}

Approval was obtained through the institutional review board for this single institution, retrospective review of patients with acute patellofemoral dislocation between January 1, 1998, and December 31, 2010. A search of our institution's database was performed, using keywords "patellofemoral subluxation/dislocation/instability" or "displaced patella." This generated a list of 2,039 patients. Clinical records were reviewed and selected for inclusion based on the following criteria:

- No prior history of patellofemoral subluxation/dislocation of the affected knee.

- X-rays within 4 weeks of the initial instability episode.

- A dislocated patella requiring reduction, or convincing history/findings suggestive of acute patellar dislocation (a subluxation or dislocation event associated with full giving away, effusion/hemarthrosis, tenderness along the medial parapatellar structures, and apprehension with lateral patellar translation). ${ }^{1,3,13}$

Patients were excluded for the following reasons: associated major ligamentous injury (anterior cruciate ligament, posterior cruciate ligament, or lateral collateral ligament) or knee dislocation (115 patients), chronic/recurrent instability at the time of presentation or previous patellofemoral disorder (i.e., patellofemoral pain syndrome) (973), lack of appropriate imaging/records (55), history of total knee arthroplasty (15), no clear history of acute patellofemoral dislocation (566), or no research authorization (3).

Therefore, 1,727 patients were excluded from the initial 2,039 in the database. This left 312 patients, including 14 with bilateral involvement, for a total of 326 knees. Demographic data were recorded and radiographs were reviewed.

Of note, the results of the pediatric/adolescent portion of this cohort were previously reported. ${ }^{14}$

\section{Radiographic Measurements}

Radiographs performed within 4 weeks of the initial injury were obtained and analyzed by the senior authors (A.M., D.D.), who were blinded to the clinical history. Lateral and merchant view radiographs were evaluated for trochlear dysplasia using the Dejour classification system. ${ }^{8,10}$ Severity was graded based on the presence of the following: crossing sign, supratrochlear spur, and/or double contour sign. ${ }^{8,10}$

Patella alta was assessed on lateral radiographs using the Caton-Deschamps index and the Insall-Salvati index..$^{15,16}$ If the patient met the definition of patella alta based on either index $(>1.2)$, then he/she was considered to have patella alta (i.e., did not require both). Skeletal maturity was evaluated based on the distal femoral and proximal tibial physes, using one of the following categories: open, closing, or closed. Patients with open or closing physes were considered skeletally immature, and those with closed physes were considered skeletally mature. ${ }^{17}$

\section{Statistical Methods}

Statistical analysis was performed by a certified statistician, using SAS software. Pearson chi-square test was used to determine differences in demographic variables. The association of patient characteristics with the risk of recurrence was assessed using Cox proportional hazards regression, accounting for correlated data within patients having bilateral patellar dislocations. Recurrence-free survival was estimated with the Kaplan-Meier method. A p-value of $<0.05$ was considered statistically significant, with a $95 \%$ confidence interval.

\section{Results}

There were 326 knees (312 patients) who met criteria for inclusion ( - Table 1). There were 145 females (46.5\%) and 167 males (53.5\%), with an average age of 19.6 years (range, 9-62 years). There were 55 patients (18.8\%) whose occupation involved manual labor, and 238 students (81.2\%). One hundred ninety-seven $(60.6 \%)$ were sports-related injuries. One hundred sixteen knees (35.7\%) demonstrated trochlear dysplasia, and $132(40.6 \%)$ had patella alta. Of those with trochlear dysplasia, 63 (54.3\%) were male and 53 (45.7\%) were female. One hundred fifteen knees (35.3\%) were skeletally immature, defined as open (8.6\%) or closing (26.7\%) physes, and 211 (64.7\%) were skeletally mature, defined as completely closed physes.

Of the total 326 cases of acute patellar dislocation, 35 (10.7\%) underwent early surgery ( - Table 2 ). The mean time to surgery was 37 days. Of these 35 patients, 8 (22.9\%) went 
Table 1 Demographics

\begin{tabular}{|l|l|}
\hline$N=326$ knees & No. (\%) \\
\hline \multirow{2}{*}{ Gender ( $N=312$ patients) } & Females: 145 (46.5\%) \\
\cline { 2 - 2 } & Males: 167 (53.5\%) \\
\hline \multirow{2}{*}{ Age $(y)$} & Average: 19.6 \\
\cline { 2 - 2 } & Range: $9-62$ \\
\hline Occupation ${ }^{a}$ & Manual labor: $55(18.8 \%)$ \\
\cline { 2 - 2 } & Student: $238(81.2 \%)$ \\
\hline Sports-related injuries & 197 (60.4\%) \\
\hline Trochlear dysplasia & $116(35.6 \%)$ \\
\hline Patella alta & 132 (40.5\%) \\
\hline Skeletal maturity & Open physes: $28(8.6 \%)$ \\
\cline { 2 - 2 } & Closing physes: 87 (26.7\%) \\
\cline { 2 - 2 } & Closed physes: $211(64.7 \%)$ \\
\hline
\end{tabular}

anformation regarding occupation was not available for 33 patients.

Table 2 Early surgical intervention

\begin{tabular}{|l|l|l|l|}
\hline & & \multicolumn{2}{l|}{$\begin{array}{l}\text { Recurrent instability } \\
\text { (no.) }\end{array}$} \\
\hline Operation & No. (total 35) & Yes & No \\
\hline Arthroscopy, loose body removal & 17 & 4 & 13 \\
\hline Arthroscopy, loose body removal, MPFL repair & 10 & 3 & 7 \\
\hline Arthroscopy, loose body removal, MPFL reconstruction & 1 & 0 & 1 \\
\hline Arthroscopy, loose body removal, lateral retinacular release & 2 & 1 & 1 \\
\hline Arthroscopy, loose body removal, medial patellar chondroplasty & 1 & 0 & 1 \\
\hline MPFL repair, open patellar realignment & 1 & 0 & 1 \\
\hline MPFL repair & 3 & 0 & 3 \\
\hline
\end{tabular}

Abbreviation: MPFL, medial patellofemoral ligament.

on to have recurrent instability, and 7 of these 8 (87.5\%) required a second or third surgery to gain stability.

The remaining patients were initially treated nonoperatively. Of the 291 patients in this group, 89 (30.6\%) had recurrent instability. For 77 (86.5\%) of these 89 patients, a recurrent instability episode occurred within the first 3 years. Forty-four (49.4\%) of these 89 patients later required operative management.

When considering the group as a whole (regardless of initial treatment), 97 (29.8\%) of 326 cases had recurrent patellofemoral subluxation/dislocation during the available follow-up period. The average time to recurrence was 16.5 months. Fifty-four (55.7\%) were male and 43 (44.3\%) were female. Fifty-six (57.7\%) of the 97 had trochlear dysplasia, and 51 of the 97 (52.6\%) underwent subsequent surgical treatment. The remaining 46 patients declined surgical intervention.

Demographic variables were analyzed independently to determine associations with increased risk of recurrent in- stability (-Table 3). Several risk factors were identified. Recurrent instability events were associated with younger age (hazard ratio [HR], 1.09; 95\% CI, 1.05-1.13; $p<0.01$ ), immature physes (HR, 2.22; 95\% CI, 1.45-3.41; $p<0.01$ ), a sports-related mechanism of injury (HR, 1.97; 95\% CI, 1.24$3.12 ; p<0.01$ ), trochlear dysplasia (HR, 3.27; 95\% CI, 2.144.99; $p<0.01$ ), and patella alta (HR, 1.61; 95\% CI, 1.07-2.43; $p=0.02$ ).

In this series, gender ( $\mathrm{HR}, 0.87 ; 95 \% \mathrm{CI}, 0.57-1.32$; $p=0.51$ ) and BMI (HR, 0.98; 95\% CI, 0.82-1.17; $p=0.8$ ) were not statistically associated with recurrent instability.

We also examined these variables in combination, specifically the presence or absence of patella alta and trochlear dysplasia, as well as age. The 5-year risk of recurrence estimates are shown in -Table 4.

Of the total 291 patients in this series, 276 (95\%) were seen in follow-up. The mean follow-up time in this series was 3.2 years (range, $0-13.5$ years). For statistical purposes, follow-up was terminated when a recurrent instability event occurred. 
Table 3 Kaplan-Meier estimates and associations with risk of recurrence

\begin{tabular}{|l|l|l|l|l|l|l|}
\hline Variable & Levels & Total (event) & $\begin{array}{l}\text { 2-y recurrence free } \\
\text { estimates (95\% Cl) }\end{array}$ & $\begin{array}{l}5-y \text { recurrence free } \\
\text { estimates (95\% Cl) }\end{array}$ & $\begin{array}{l}\text { Hazard ratio } \\
(95 \% \text { CI) }\end{array}$ & $p$ Value \\
\hline Gender & Male & $155(49)$ & $71 \%(63,79)$ & $57 \%(48,68)$ & $1.0($ reference) & - \\
\hline & Female & $136(40)$ & $74 \%(66,82)$ & $66 \%(58,76)$ & $0.87(0.57,1.32)$ & 0.51 \\
\hline Age, per year & - & $291(89)$ & - & - & $1.09(1.05,1.13)$ & $<0.01$ \\
\hline BMI & - & $184(72)$ & & & $0.98(0.82,1.17)$ & 0.80 \\
\hline Occupation & Student/other & $260(88)$ & $70 \%(64,76)$ & $59 \%(52,66)$ & $1.0($ reference) & - \\
\hline & Laborer & $15(1)$ & $100 \%(100,100)$ & $86 \%(63,100)$ & $0.16(0.03,1.08)$ & 0.06 \\
\hline $\begin{array}{l}\text { Mechanism of } \\
\text { injury }\end{array}$ & Nonsport & $119(24)$ & $82 \%(74,90)$ & $76 \%(67,85)$ & 1.0 (reference) & - \\
\hline & Sport & $171(64)$ & $67 \%(59,75)$ & $53 \%(45,63)$ & $1.97(1.24,3.12)$ & $<0.01$ \\
\hline Patella alta & No & $173(45)$ & $77 \%(71,84)$ & $68 \%(60,77)$ & $1.0($ reference) & - \\
\hline & Yes & $117(43)$ & $66 \%(57,76)$ & $53 \%(43,65)$ & $1.61(1.07,2.43)$ & 0.02 \\
\hline Physeal status & Mature & $187(39)$ & $78 \%(72,85)$ & $74 \%(67,82)$ & $1.0($ reference) & - \\
\hline & Immature & $104(50)$ & $63 \%(54,74)$ & $44 \%(35,57)$ & $2.22(1.45,3.41)$ & $<0.01$ \\
\hline $\begin{array}{l}\text { Trochlear } \\
\text { dysplasia }\end{array}$ & No & $187(36)$ & $82 \%(76,89)$ & $75 \%(68,83)$ & $1.0($ reference) & - \\
\hline & Yes & $103(52)$ & $56 \%(47,68)$ & $40 \%(30,52)$ & $3.27(2.14,4.99)$ & $<0.01$ \\
\hline
\end{tabular}

Abbreviation: BMI, body mass index.

Table 4 Risk of recurrence for combined variables

\begin{tabular}{|l|l|l|l|}
\hline Patella alta & Trochlear dysplasia & Younger than 25 y & $5-y$ risk of recurrence (95\% Cl) \\
\hline+ & + & + & $70.4 \%(54 \%, 80.9 \%)$ \\
\hline- & + & + & $60.2 \%(44.4 \%, 71.5 \%)$ \\
\hline+ & - & + & $33.6 \%(20.8 \%, 44.3 \%)$ \\
\hline+ & + & - & $29.6 \%(6.7 \%, 46.9 \%)$ \\
\hline- & - & + & $26.6 \%(17.1 \%, 35.1 \%)$ \\
\hline- & + & - & $23.3 \%(5.2 \%, 38 \%)$ \\
\hline+ & - & - & $11.1 \%(2.1 \%, 19.3 \%)$ \\
\hline- & - & - & $8.6 \%(1.8 \%, 14.8 \%)$ \\
\hline
\end{tabular}

Therefore, the median follow-up was 4.0 years, with 147 (73\%) of 202 patients having greater than 1 year of follow-up.

Patients were categorized based on age (greater or less than 25 years) and trochlear dysplasia (present or absent).
Compared with other groups, patients under 25 years of age with trochlear dysplasia had an HR of 3.06 and a statistically significant $p$-value $(<0.01)$ (-Table 5). The 2-year KaplanMeier recurrence-free estimate for this group was $51 \%$. This is

Table 5 Analysis of maximum likelihood estimates-age and trochlear dysplasia

\begin{tabular}{|l|l|l|}
\hline & Hazard ratio $(95 \% \mathrm{Cl})$ & $p$ Value \\
\hline$<25$ y old and no trochlear dysplasia & 1.0 (reference) & - \\
\hline$<25$ y old and trochlear dysplasia & $3.06(1.95-4.78)$ & $<0.01$ \\
\hline$>25$ y old and no trochlear dysplasia & $0.28(0.09-0.88)$ & 0.03 \\
\hline$>25$ y old and trochlear dysplasia & $0.91(0.30-2.71)$ & 0.86 \\
\hline
\end{tabular}




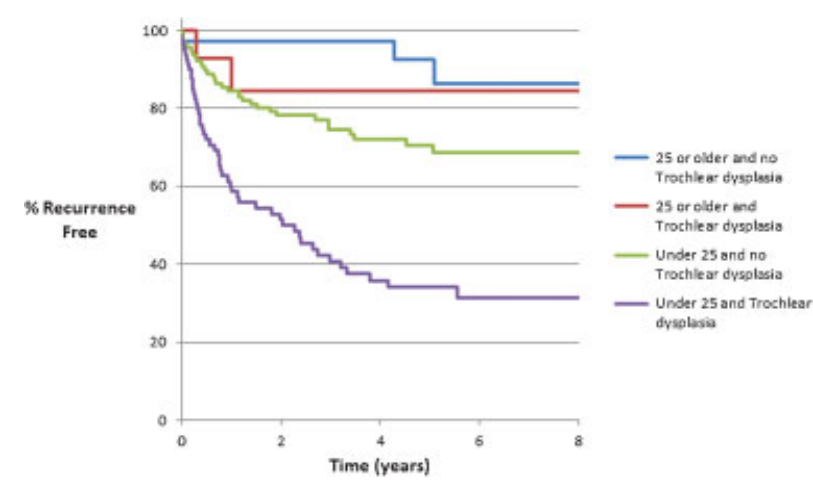

Fig. 1 Recurrence-free survival estimates-age and trochlear dysplasia.

compared with that of patients older than 25 years without trochlear dysplasia, for whom the 2-year Kaplan-Meier recurrence-free estimate was $97 \%$ (-Fig. 1).

Patients were categorized based on the presence or absence of trochlear dysplasia and patella alta. Trochlear dysplasia and patella alta were again identified as a statistically significant risk factors, as shown in - Table 6. The 2-year Kaplan-Meier recurrence-free estimate for patients with trochlear dysplasia and patella alta was $48 \%$, compared with $83 \%$ for those without either (-Fig. 2). The 5-year estimates were 38 and $80 \%$, respectively.

\section{Discussion}

In this study, we describe the epidemiology of acute patellar dislocation and identify risk factors for recurrent instability. Strengths of this study include its large numbers, strict inclusion criteria, and radiographic evaluation. Limitations of this study include its retrospective nature and incomplete followup. In addition, magnetic resonance imaging was not routinely performed to radiographically confirm the diagnosis of a lateral patellar dislocation. Mechanical axis/limb alignment and tibial tubercle to trochlear groove (TT-TG) distance could not be evaluated as standing anterior/posterior lower extremity full-length radiographs and axial CT were not performed on all patients.

To our knowledge, this is one of the largest series of acute patellar dislocations in the literature. Hsiao et al conducted an epidemiologic study of more than 9,000 military service members with documented patellar dislocation injuries, and found the highest incidence of acute dislocations in the

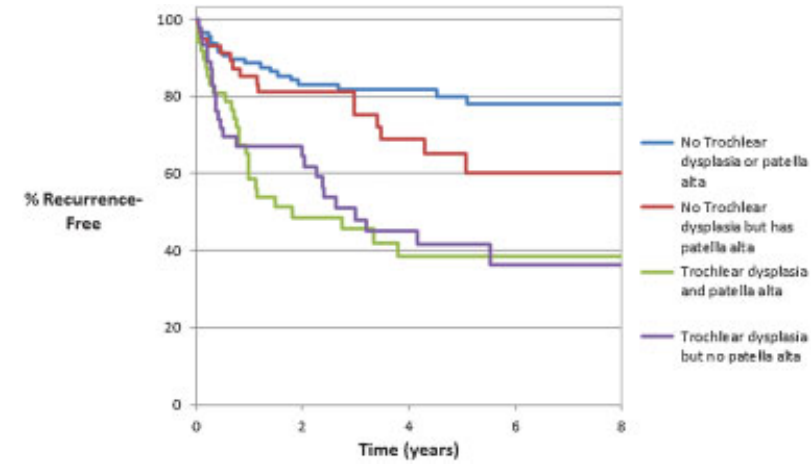

Fig. 2 Recurrence-free survival estimates-trochlear dysplasia and patella alta.

youngest age group. ${ }^{18}$ Service members under 20 years of age were $84 \%$ more likely to sustain these injuries compared with those over 40 years of age. These authors did not evaluate recurrent instability. Fithian et al performed a large study, comparing 125 patients with a first-time dislocation to 64 patients with recurrent dislocations. ${ }^{3}$ Most other studies on this topic have been smaller in size, with fewer than 100 patients. ${ }^{19,20}$

In our series, approximately $10 \%$ of patients were initially treated with surgery after the first dislocation. This is consistent with previous reports by Nietosvaara et $\mathrm{al}^{4}$ and others. These patients had a $22.9 \%$ rate of recurrent instability. The reason for this rather high rate of recurrence may be that most did not undergo acute stabilizing procedures. Twenty patients underwent arthroscopy/loose body removal alone, without any type of stabilizing procedure. Of those, five went on to exhibit recurrent instability. Fifteen patients underwent some form of acute stabilization. Of those, three went on to recurrent instability (-Table 2 ).

When analyzing the entire group (regardless of initial treatment), approximately $30 \%$ of patients had recurrent instability, and half underwent subsequent surgical intervention. Recurrent instability was more likely in younger, skeletally immature patients, with sports-related injuries, and those with radiographic evidence of patella alta and trochlear dysplasia.

Controversy exists as to whether gender influences one's risk for patellar dislocation. Some authors have found an increased incidence among females, ${ }^{4}$ though others have questioned whether this holds true. ${ }^{3}$ Atkin et al found an equal distribution among 74 men and women with acute

Table 6 Analysis of maximum likelihood estimates-trochlear dysplasia and patella alta

\begin{tabular}{|l|l|l|}
\hline & Hazard ratio $(95 \% \mathrm{Cl})$ & $p$ Value \\
\hline- TD, - PA & 1.0 (reference) & \\
\hline- TD, + PA & $1.69(0.88-3.26)$ & 0.12 \\
\hline+ TD, -PA & $3.73(2.05-6.76)$ & $<0.01$ \\
\hline+ TD, +PA & $4.29(2.39-7.7)$ & $<0.01$ \\
\hline
\end{tabular}

Abbreviations: PA, patella alta; TD, trochlear dysplasia. 
dislocations. ${ }^{1}$ In our series, the gender distribution was nearly equal, and did not play a significant role in the risk for recurrent instability. Interestingly, females accounted for slightly less than half $(45.7 \%)$ of patients with trochlear dysplasia.

Older age was found to be protective in our study. With each one year age increase, the risk of recurrent instability decreased by approximately $8 \%$. Along the same lines, skeletally immature patients had greater than two times the risk of recurrent instability as those who were skeletally mature. This is to be expected, given the association between age and physeal status. Of note, none of the patients over 40 years were found to have a repeat instability event (-Fig. 3).

The mechanism of injury also had implications for recurrent episodes of instability. Patients involved in sports at the time of the initial dislocation were nearly twice as likely to have ongoing instability as those not involved in sports. This is consistent with previous studies, ${ }^{2,3}$ and may be related to the large number of patients returning to high-risk activities.

Patients (of any age) with trochlear dysplasia had the greatest risk of recurrent instability, which was three times greater than those without trochlear dysplasia. This is clinically relevant information, which is helpful when counseling patients on treatment goals. Nearly $58 \%$ of recurrences in this series were found to have trochlear dysplasia. The combination of trochlear dysplasia and younger age demonstrated a three times increased risk of recurrent instability. Nearly half of these patients exhibited recurrent instability within the first two years. The combination of trochlear dysplasia and patella alta increased risk by 4.2 times.

Earlier surgical treatment may be recommended for patients with trochlear dysplasia who are younger than age $25(+/-$ patella alta), given the 60 to $70 \%$ risk of recurrence by 5 years. This is compared with patients of the same age without trochlear dysplasia or patella alta, for whom the 5 -year risk of recurrence is only $27 \%$. The chart shown in -Fig. 4 may be a useful clinical tool when counseling patients and their families.

In this large series, patients with a first-time patellofemoral dislocation had a 30\% recurrence rate. Younger age, immature physes, sports-related injuries, patella alta, and trochlear dysplasia were all associated with an increased risk of recurrent

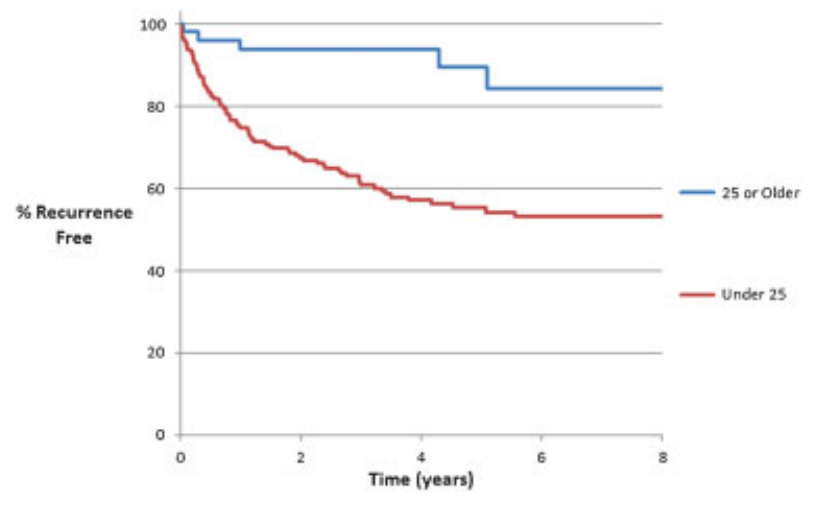

Fig. 3 Recurrence-free survival estimates-age.

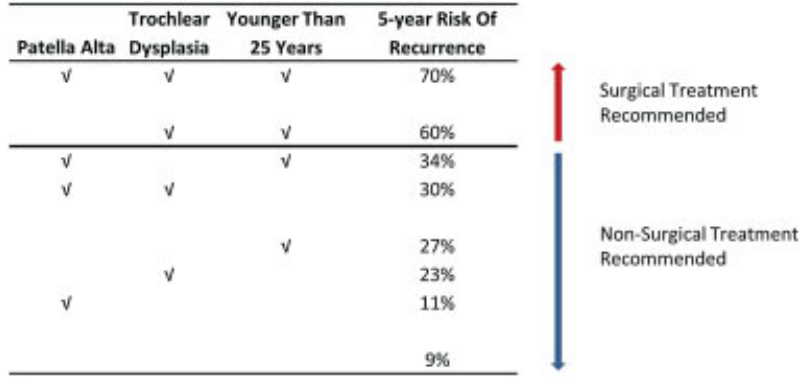

Fig. 4 Clinical tool to assess risk of recurrence.

instability events. In particular, patients with both trochlear dysplasia and immature physes, as well as trochlear dysplasia and patella alta, resulted in the highest risk of recurrent instability. These factors should be considered when making decisions regarding operative management and timing.

\section{References}

1 Atkin DM, Fithian DC, Marangi KS, Stone ML, Dobson BE, Mendelsohn C. Characteristics of patients with primary acute lateral patellar dislocation and their recovery within the first 6 months of injury. Am J Sports Med 2000;28(4):472-479

2 Buchner M, Baudendistel B, Sabo D, Schmitt H. Acute traumatic primary patellar dislocation: long-term results comparing conservative and surgical treatment. Clin J Sport Med 2005;15(2):62-66

3 Fithian DC, Paxton EW, Stone ML, et al. Epidemiology and natural history of acute patellar dislocation. Am J Sports Med 2004;32(5): 1114-1121

4 Nietosvaara Y, Aalto K, Kallio PE. Acute patellar dislocation in children: incidence and associated osteochondral fractures. J Pediatr Orthop 1994;14(4):513-515

5 Shea KG, Grimm NL, Belzer J, Burks RT, Pfeiffer R. The relation of the femoral physis and the medial patellofemoral ligament. Arthroscopy 2010;26(8):1083-1087

6 Arendt EA, Fithian DC, Cohen E. Current concepts of lateral patella dislocation. Clin Sports Med 2002;21(3):499-519

7 Stefancin JJ, Parker RD. First-time traumatic patellar dislocation: a systematic review. Clin Orthop Relat Res 2007;455(455):93-101

8 Dejour D, Le Coultre B. Osteotomies in patello-femoral instabilities. Sports Med Arthrosc Rev 2007;15(1):39-46

9 Cash JD, Hughston JC. Treatment of acute patellar dislocation. Am J Sports Med 1988;16(3):244-249

10 Bollier M, Fulkerson JP. The role of trochlear dysplasia in patellofemoral instability. J Am Acad Orthop Surg 2011;19(1):8-16

11 Cofield RH, Bryan RS. Acute dislocation of the patella: results of conservative treatment. J Trauma 1977;17(7):526-531

12 Hawkins RJ, Bell RH, Anisette G. Acute patellar dislocations. The natural history. Am J Sports Med 1986;14(2):117-120

13 Mäenpää H, Lehto MU. Patellar dislocation. The long-term results of nonoperative management in 100 patients. Am J Sports Med 1997;25(2):213-217

14 Lewallen LW, McIntosh AL, Dahm DL. Predictors of recurrent instability after acute patellofemoral dislocation in pediatric and adolescent patients. Am J Sports Med 2013;41(3):575-581

15 Dejour H, Walch G, Nove-Josserand L, Guier C. Factors of patellar instability: an anatomic radiographic study. Knee Surg Sports Traumatol Arthrosc 1994;2(1):19-26

16 Thévenin-Lemoine C, Ferrand M, Courvoisier A, Damsin JP, Ducou le Pointe $\mathrm{H}$, Vialle R. Is the Caton-Deschamps index a valuable ratio to investigate patellar height in children? J Bone Joint Surg Am 2011;93(8):e35 
17 McCarroll JR, Shelbourne KD, Porter DA, Rettig AC, Murray S. Patellar tendon graft reconstruction for midsubstance anterior cruciate ligament rupture in junior high school athletes. An algorithm for management. Am J Sports Med 1994;22(4):478-484

18 Hsiao M, Owens BD, Burks R, Sturdivant RX, Cameron KL. Incidence of acute traumatic patellar dislocation among active-duty United States military service members. Am J Sports Med 2010;38(10): 1997-2004
19 Balcarek P, Ammon J, Frosch S, et al. Magnetic resonance imaging characteristics of the medial patellofemoral ligament lesion in acute lateral patellar dislocations considering trochlear dysplasia, patella alta, and tibial tuberosity-trochlear groove distance. Arthroscopy 2010;26(7):926-935

20 Lippacher S, Reichel H, Nelitz M. Radiological criteria for trochlear dysplasia in children and adolescents. J Pediatr Orthop B 2011; 20(5):341-344 